\title{
Mammotome: Less Invasive than ABBI with Similar Accuracy for Early Breast Cancer Detection
} Walter P. Weber, M.D., ${ }^{1}$ Rosanna Zanetti, M.D., ${ }^{2}$ Igor Langer, M.D., ${ }^{1}$ Sophie Dellas, M.D., ${ }^{3}$ Markus Zuber, M.D., ${ }^{5}$
Holger Moch, M.D., ${ }^{4}$ Eugenia Remmel, M.D., ${ }^{6}$ Daniel Oertli, M.D., ${ }^{1}$ Edward Wight, M.D.,, Walter R. Marti, M.D.

${ }^{1}$ Department of Surgery, University of Basel, Spitalstrasse 21, Basel, CH-4031 Switzerland

${ }^{2}$ Department of Gynaecology, University of Basel, Spitalstrasse 21, Basel, CH-4031 Switzerland

${ }^{3}$ Department of Radiology, University of Basel, Spitalstrasse 21, Basel, CH-4031 Switzerland

${ }^{4}$ Department of Pathology, University of Basel, Spitalstrasse 21, Basel, CH-4031Switzerland

${ }^{5}$ Department of Surgery, Regional Hospital of Olten, Baslerstrasse 150, Olten, Ch-4600 Switzerland

${ }^{6}$ Department of Gynaecology, University of Ulm, Prittwitzstrasse 43, Ulm, D-89075 Germany

Published Online: March 22, 2005

\begin{abstract}
We performed a prospective analysis of two consecutive biopsy cohorts investigated by the same team to compare the Mammotome system with the ABBI procedure. From April 1997 to August 2003 a series of 413 nonpalpable mammographic lesions in 387 women (median age 56 years, range 30-84 years) were stereotactically biopsied in the University Hospital of Basel, Switzerland. Until October 1999 the ABBI system was applied exclusively, it was subsequently superseded by the Mammotome device in our clinic. Main outcome measures were accuracy, technical demand, and morbidity. Sensitivity $(\mathbf{9 7 . 3} \% / 96.8 \%)$, negative predictive value $(99.2 \% / 98.7 \%)$, and diagnostic accuracy $(99.4 \% / 99.1 \%)$ regarding the detection of malignancy were excellent for both techniques (ABBI/ Mammotome). The Mammotome procedure was faster and less invasive, thus causing significantly less morbidity. The larger specimen obtained by the ABBI procedure resulted in more detailed histology. In conclusion, recommend the Mammotome system as the method of choice for detecting nonpalpable early breast cancer.
\end{abstract}

The biopsy of nonpalpable suspicious radiopaque breast lesions is a challenging procedure carried out by mammographically or sonographically guided techniques. The advanced breast biopsy instrumentation (ABBI; United States Surgical Corporation, Norwalk, CT, USA) as one of the first stereotactically imaging instruments has been established with high accuracy [1-11]. Disadvantages of this method are its time-consuming complexity and the residual scar, with a length of 10 to $30 \mathrm{~mm}$ depending on the caliber of the cutting cannula used. Subsequently, the stereotactic vacuum-assisted core breast biopsy (Mammotome, Ethicon EndoSurgery, Cincinnati, OH, USA) has successfully been introduce $[11,17-17]$. The aim of this study was to assess the Mammotome system and to compare its accuracy, technical demand, and morbidity to that of the ABBI procedure performed in a consecutive series of patients by the same investigating team.

Correspondence to: Walter R. Marti, M.D., e-mail: wrmarti@uhbs.ch

\section{Patients and Methods}

From April 1997 we entered the histologic findings and follow-up data of all patients with an ABBI biopsy in our hospital prospectively into a spreadsheet database (Excel, Microsoft). The Mammotome system was introduced in our clinic during October 1999. After an initial training period of 5 months during which still 19 ABBI procedures were performed, all stereotactically guided biopsies were carried out using the Mammotome system. Consecutive data from patients biopsied until August 2003 were included in the study. Indications for a stereotactic biopsy were mammographically suspicious, but impalpable breast lesions according to BIRADS III-V. There was no further selection of patients. The ABBI procedure was performed as earlier described $[1,5,8,18,19-5,8,18,19]$. Its oscillating blade-cutting cannula produces one large cylinder of the suspicious breast tissue with sharp margins. These excisional biopsies and the following documentation of data were performed by the same team of experienced surgeons and gynecologists with their registrars in our hospital.

The Mammotome technique, as previously described [2, 12, 16], using the same stereotactically guided interventionist table (Lorad stereoguide table; LoRad, Danbury, CT, USA) achieves a representative specimen by multiple tissue cores obtained by vacuum suction in a circumferential manner. Therefore an 11-gauge biopsy needle is inserted under stereotactic guidance. Because the Mammotome allows a less invasive procedure to be performed, a few specialized radiologists joined our interventional diagnostic team.

During all stereotactic procedures the site of lesion was marked with a metal clip to allow easy relocalization for further treatment if required. Removal of the suspicious lesion was documented by radiography of the resected specimen in combination with a stereotactic mammogram of the excisional site immediately after the biopsy was performed. All histologic diagnoses were confirmed by a second pathologist. The incision after ABBI procedures was 
Table 1. Patients and technical data.

\begin{tabular}{lll}
\hline Parameter & ABBI & Mammotome \\
\hline Study period & April 1997 to March 2000 & October 1999 to August 2003 \\
Median age (years) & $56.2(33-78)(159$ patients) & $56.1(30-84)(228$ patients) \\
Nonpalpable, suspicious lesions $(\mathrm{n}=413)$ & 174 & 239 \\
Successful biopsies & $162(93 \%)$ & $223(93 \%)$ \\
Breast too small/lesion too close to the chest wall & $5(2.9 \%)$ & $5(2.1 \%)$ \\
Lesion not evident on image & $5(2.9 \%)$ & $8(3.3 \%)$ \\
Biopsy refused/discontinuation because of pain & $2(1.1 \%)$ & $1(0.4 \%)$ \\
Technical problems & 0 & $2(0.8 \%)$ \\
Median duration (minutes)* & $90(50-180)$ & $60(30-150)$ \\
\hline
\end{tabular}

Demographic and technical data of 413 Consecutive Stereotacric breast biopsies in 387 women $* p<0.0001$.

closed by interrupted sutures, and the stab incision after Mammotome biopsies was closed simply by SteriStrips. A compressive bandage left for 24 hours to prevent excessive hematoma and removal of the skin sutures or SteriStrips after 5 days combined with a discussion of the histologic findings completed the investigation. All stereotactic biopsies were performed under local anesthesia on an outpatient basis.

Whenever possible, a malignant lesion was treated by breastconserving tumorectomy followed by adjuvant radiotherapy and systemic therapy if indicated. Patients with benign lesions were followed according to the referring physician's concept of optimal prevention appropriate to the individual risk for breast cancer. Women with benign lesions and a low risk were routinely advised to undergo clinical examination and mammography with or without sonography every other year on a screening basis from age 50 up to the age of 69 . The patient's histopathologic findings and follow-up data after definitive treatment of malignant findings were entered prospectively into a database (Excel, Microsoft Access Database Software; Microsoft, Redmond, WA, USA). Follow-up data for patients with benign lesions were obtained from the patients themselves and their attending physicians. Morbidity was assessed using a standardized questionnaire. We addressed the patient's subjective experience during the investigation, followed by a series of questions pointing at wound healing and their long-term course.

The diagnostic characteristics of the two techniques were evaluated by compariaon of the biopsy results with the definitive pathologic diagnosis and by analyzing the follow-up data on benign lesions. Mammographic/sonographic examination were performed in $93 \%(111 / 120) / 88 \%(106 / 120)$ of the patients with a benign ABBI result, whereas $78 \%(117 / 150) / 76 \%$ (114/150) of the patients in the Mammotome cohort have had at least one radiologic reassessment of the breast since the biopsy. Most of the patients without imaging follow-up are young women with low risk for breast cancer not yet qualifying for screening mammography. All patients had at least one clinical investigation of the breast since the biopsy, the latest after a median follow-up of 5.2 years (range 3.8-6.7 years) for the ABBI cohort and 2.1 years (range 0.5-4.4 years) for the Mammotome series. Follow-up data on an additional 5 benign ABBI biopsies and 12 benign Mammotome biopsies were not available for analysis.

The unpaired $t$-test was used for comparisons of continuous outcomes, and Fisher's exact test was used for comparisons of dichotomous and categoric variables. Statistical significance was defined below an $\alpha$ level of $p=0.05$. All statistical tests were twosided. Statistical analyses were performed by GraphPad InStat software version 3.05 (GraphPad Software, San Diego, CA, USA).

\section{Results}

Indications for 413 consecutive stereotactic breast biopsies in 387 women were 331 cases of microcalcifications, 61 nodular masses, and 21 other mammographically suspicious and nonpalpable lesions. Irrespective of the technique applied, 93\% of the biopsies were performed successfully (Table 1). The median duration of the Mammotome procedure was significantly shorter $(p<$ $0.0001)$.

Most of the biopsy procedures were well tolerated in both series. However, a small number of patients complained about severe pain despite further injection of local anesthetic (seven patients in each cohort, including two for procedures that had to be interrupted because of pain in the ABBI series and one in the Mammotome group). Three patients were treated by both techniques. They mentioned no preferences for one or the other, and the durations of the two procedures were comparable.

Surgical complications were rare and mild (Table 2). The morbidity rate after a Mammotome biopsy was significantly lower than after an ABBI procedure $(p<0.022)$. The scar was readily accepted by most of the patients.

The rate of malignancy was comparable in the two groups and within the range of various recently published consecutive series of stereotactic breast biopsies [3, 9, 11, 15, 17]. The detected histologic findings are outlined in Table 3.

Sensitivity, negative predictive value, and diagnostic accuracy were excellent for both types of biopsy (Table 4). One invasive lobular carcinoma was sonographically detected in the clipmarked area of the breast 3 months after a false-negative ABBI biopsy that revealed nonsuspicious tissue. In the Mammotome series two malignant lesions were missed: In the first case the biopsy of suspicious microcalcification demonstrated fibrotic changes, but the presence of only minimal calcareous traces on radiography of the resected specimen initiated further investigations. After 4 months mammography showed virtually unchanged microcalcification compared to the prebiopsy image. A second Mammotome procedure resulted in the diagnosis of a low-grade ductal carcinoma in situ (DCIS) treated by tumorectomy; a third Mammotome biopsy in the same breast 1 year later uncovered a multicentric DCIS, and finally a mastectomy was necessary. In the second case the patient had breast-conserving therapy and axillary treatment of an invasive carcinoma (pT2pN1cM0 G3 R0) followed by bilateral reduction mammoplasty for cosmetic reasons. 
Table 2. Morbidity.

\begin{tabular}{|c|c|c|c|}
\hline Morbid condition & $\mathrm{ABBI}(n=120 / 125)^{a}$ & $p$ & Mammotome $(n=150 / 162)^{a}$ \\
\hline Wound infection & $1(0.8 \%)$ & 1.00 & $2(1.3 \%)$ \\
\hline Skin lesion & $2(1.7 \%)$ & 0.20 & 0 \\
\hline Severe bruising/seromat ${ }^{b}$ & $5(4.2 \%)$ & 0.76 & $5(3.3 \%)$ \\
\hline Persistent pain/discomfort & $4(3.3 \%)$ & 0.41 & $2(1.3 \%)$ \\
\hline Incomplete satisfaction with the cosmetic result & $8(6.7 \%)$ & 0.03 & $2(1.3 \%)$ \\
\hline Total & $20(16.7 \%)$ & 0.02 & $11(7.3 \%)$ \\
\hline
\end{tabular}

Morbidity was assessed by collecting the follow-up data of 270 patients with benign lesions and no further surgical intervention of the breast from the patients themselves and their attenting physicians using a standardized questionnaire.

Overall morbidity was significantly less in the Mammotome series $(\mathrm{p}=0.021$, Fisher's exact test).

${ }^{a}$ Follow-up data of 5 additional benign ABBI and 12 benign Mammotome biopsies were not available for analysis.

${ }^{b}$ Requiring interventional therapy or conservative treatment over a period of several months.

Table 3. Histopathologic findings.

\begin{tabular}{|c|c|c|}
\hline Histopathologic finding & $\mathrm{ABBI}(n=162)$ & Mammotome $(n=223)$ \\
\hline Malignant lesions & $37(22.8 \%)$ & $61(27.4 \%)$ \\
\hline \multicolumn{3}{|l|}{ Invasive cancer } \\
\hline Ductal only & 3 & 12 \\
\hline Ductal and ductal in situ & 8 & 6 \\
\hline Lobular only & 1 & 1 \\
\hline Lobular and lobular or ductal in situ & 1 & 1 \\
\hline Tubular with or without ductal in situ & 3 & 1 \\
\hline Mucinous & 1 & - \\
\hline \multicolumn{3}{|l|}{ Carcinoma in situ } \\
\hline Ductal only & 17 & 40 \\
\hline Ductal and lobular & 3 & - \\
\hline Benign lesions ${ }^{a}$ & $125(77.2 \%)$ & $162(72.6 \%)$ \\
\hline Mastopathy/benign proliferative changes & 81 & 93 \\
\hline Fibrosis/scar tissue & 24 & 45 \\
\hline Fibroadenoma & 13 & 15 \\
\hline \multicolumn{3}{|l|}{ Borderline lesions } \\
\hline Papilloma & 1 & 1 \\
\hline Atypical ductal or lobular hyperplasia/lobular carcinoma in situ & 6 & 8 \\
\hline
\end{tabular}

Analysis of the histopathologic findings of all 385 successfully performed stereotactic breast biopsies.

${ }^{a}$ The main component of mixed benign lesions was recorded.

Table 4. Characteristics of Mammotome versus ABBI regarding the detection of malignancy.

\begin{tabular}{|c|c|c|c|}
\hline Characteristic & $\mathrm{ABBI}(n=157)^{a}$ & $p$ & Mammotome $(n=211)^{a}$ \\
\hline Sensitivity & $97.3 \%(37 / 38)$ & & $96.8 \%(61 / 63)$ \\
\hline Negative predictive value & $99 . \%(119 / 120)$ & & $98.7 \%(148 / 150)$ \\
\hline Diagnostic accuracy & $99.4 \%(156 / 157)$ & & $99.1 \%(209 / 211)$ \\
\hline In situ component missed & 3 & 1.00 & 6 \\
\hline Invasive component missed ${ }^{b}$ & 1 & 0.22 & 6 \\
\hline
\end{tabular}

The biopsy results were compared with the definitive histopathologic diagnosis and a median follow-up time for benign lesions of 2.1 years (range 0.5-4.4 years) and 5.2 years (range 3.8-6.7 years), respectively, for the Mammotome versus the ABBI techniques.

${ }^{a}$ Follow-up data of 5 additional benign ABBI and 12 benign Mammotome biopsies were not available for analysis

${ }^{b}$ Reintervention after initial surgery was necessary for axillary staging.

Four years later a Mammotome biopsy that revealed architectural and calcareous distortion in the same area of the breast showed only microcalcification and scar tissue. In a familial high-risk situation the magnetic resonance tomographic examination 4 months later was still suspicious of cancer. A wide, open biopsy proved local recurrence of the invasive carcinoma, and the procedure had to be completed with a mastectomy.

After surgical treatment we recorded a histopathologic underestimate of the malignant disease by the biopsy result in seven patients in whom the invasive component was missed by the stereotactic procedure (Table 4). Six of those biopsies uncovering only the in situ component were carried out using the incisional Mammotome technique. After tumorectomy, all seven patients had to be rescheduled for axillary staging by the sentinel lymph node procedure.

During the study period, 10 cases of atypical ductal or lobular hyperplasia (ADH/ALH) and four cases of lobular carcinoma in situ (LCIS) were observed as borderline lesions during an intensive follow-up, particularly a sonographic and clinical examinations every 6 months combined with mammography once a year. In one case the Mammotome biopsy showed ADH and ALH in combination with an LCIS. These findings in a familial 
risk situation were considered an indication for follow-up surgical excision, which revealed both a mixed invasive ductal and lobular carcinoma next to the atypical hyperplasia and LCIS. During follow-up of all other 13 atypical hyperplasia and LCIS cases, no upstaging was necessary.

\section{Discussion}

First we evaluated our ABBI system with specific regard to its potential therapeutic use [20]. The percentage of incompletely excised malignant lesions was $84 \%(26 / 31)$. The final evaluation of the ABBI series revealed that the proportion of biopsy margins infested with malignancy remained unchanged (data not shown). Several other published series showed a similar limitation $[1,6,19]$, advocating that the ABBI procedure should not be used routinely for therapeutic purposes.

Subsequently, this excisional procedure was superseded in our hospital by an even less invasive stereotactic technique, the incisional vacuum-assisted core breast biopsy (Mammotome), with the expectation of comparable diagnostic accuracy.

The identical median age of the patients and the similar rate of malignancy in our two series suggest an equal indication for the two techniques in our hospital. Thus the two series investigated by the same medical team are comparable even though the ABBI population is a historical control. Moreover, our rate of malignancy is comparable to that in several published stereotactic biopsy series $[3,9,11,15,17]$.

Our $7.1 \%(1 / 14)$ rate of upstaging $\mathrm{ADH}$ or lobular neoplasia (ALH/LCIS) detected by the Mammotome to a DCIS or an invasive carcinoma during follow-up is within the published range of $0 \%$ to $25 \%$ [21-24]. There is a common trend in the literature toward routine surgical excision of $\mathrm{ADH}$ detected by stereotactic procedures to ensure adequate staging [21-23, 25-30]; however, some authors still disagree because of the many cases of unnecessary removal of these lesions [24, 31, 32]. Likewise, the standard procedure after diagnosis of lobular neoplasia (ALH/LCIS) by the Mammotome is still controversial. Although routine surgical excision of lobular neoplasia has lately been recommended [30], most authors demand additional risk factors for a subsequent open biopsy [33-37]. In the absence of additional indications for open biopsy, such as radial scar, phylloides tumor, intraductal papilloma, or a strong personal or familial history for breast cancer, we followed patients with these borderline lesions more intensively.

The more invasive excisional ABBI technique achieved a larger specimen with sharp margins resulting in a more detailed histologic diagnosis; thus only one malignancy and one invasive component were missed in this cohort. This turned out to be the only clear advantage of the ABBI system in our comparison, although the difference was statistically not significant $(p>0.05)$. New developments such as the larger 8-gauge needle and a new 11-gauge stereo probe for the Mammotome might negate this deficiency. In contrast, the incisional Mammotome technique caused significantly less morbidity. In addition, the necessary therapeutic reexcision of the residual tunnel after an ABBI biopsy to ensure complete removal of the tumor is demanding and results in large tissue loss. Although the Mammotome is a challenging technique that requires a specialized team for an appropriate indication and precise performance, it is the less complex procedure, is more rapidly performed, and is at least equally tolerated compared to the ABBI technique. As its accuracy for the detection of malignancy is similar, we recommend the Mammotome system as the method of choice for detecting nonpalpable early breast cancer.

\section{References}

1. D'Angelo PC, Galliano DE, Rosemurgy AS. Stereotactic excisional breast biopsies utilizing the advanced breast biopsy instrumentation system. Am. J. Surg 1997;174:297-302

2. Velanovich V, Lewis FR Jr, Nathanson SD, et al. Comparison of mammographically guided breast biopsy techniques. Ann. Surg 1999;229:625-633

3. Haj M, Kniaz D, Eitan A, et al. Three years of experience with advanced breast biopsy instrumentation (ABBI). Breast J 2002;8:275280

4. Yang JH, Lee SD, Nam SJ. Diagnostic utility of ABBI ${ }^{\circledR}$ (Advanced Breast Biopsy Instrumentation) for nonpalpable breast lesions in Korea. Breast J 2000;6:257-262

5. Portincasa G, Lucci E, Navarra GG, et al. Initial experience with breast biopsy utilizing the Advanced Breast Biopsy Instrumentation (ABBI). J. Surg. Oncol 2000;74:201-203

6. Bloomston M, D'Angelo P, Galliano D, et al. One hundred consecutive advanced breast biopsy instrumentation procedures: complications, costs, and outcome. Ann. Surg. Oncol 1999;6:137-138

7. Sheth D, Wesen CA, Schroder D, et al. The advanced breast biopsy instrumentation (ABBI) experience at a community hospital. Am. Surg 1999;65:726-730

8. Matthews BD, Williams GB. Initial experience with the advanced breast biopsy instrumentation system. Am. J. Surg 1999;177:97-101

9. Insausti LP, Alberro JA, Regueira FM, et al. An experience with the Advanced Breast Biopsy Instrumentation (ABBI) system in the management of non-palpable breast lesions. Eur. Radiol 2002;12:1703-1710

10. Watermann DO, Einert A, Ehritt-Braun C, et al. Experience with the Advanced Breast Biopsy Instrumentation (ABBI) system. Anticancer Res 2002;22:3067-3070

11. Mariotti C, Feliciotti F, Baldarelli M, et al. Digital stereotactic biopsies for nonpalpable breast lesion. Surg. Endosc 2003;17:911917

12. Liberman L, Smolkin JH, Dershaw DD, et al. Calcification retrieval at stereotactic, 11-gauge, directional, vacuum-assisted breast biopsy. Radiology 1998;208:251-260

13. Klem D, Jacobs HK, Jorgensen R, et al. Stereotactic breast biopsy in a community hospital setting. Am. Surg 1999;65:737-741

14. Cangiarella J, Waisman J, Symmans WF, et al. Mammotome core biopsy for mammary microcalcification: analysis of 160 biopsies from 142 women with surgical and radiologic followup. Cancer 2001;91:173-177

15. Travade A, Isnard A, Bagard C, et al. Stereotactic 11-gauge directional vacuum-assisted breast biopsy: experience with 249 patients. J. Radiol 2002;83:1063-1071

16. Apesteguia L, Mellado M, Saenz J, et al. Vacuum-assisted breast biopsy on digital stereotaxic table of nonpalpable lesions non-recognisable by ultrasonography. Eur. Radiol 2002;12:638-645

17. Giardina C, Guerrieri AM, Ingravallo G, et al. The stereotaxic core breast biopsy using the Mammotome: an alternative to intraoperative examination. Pathologica 2002;94:182-189

18. Oertli D, Marti WR, Harder F, et al. The Advanced Breast Biopsy Instrumentation (ABBI), a system for stereotactic excision of mammographically suspect nonpalpable findings in the breast. Schweiz. Med. Wochenschr 1998;128:811-816

19. Ferzli GS, Hurwitz JB. Initial experience with breast biopsy utilizing the advanced breast biopsy instrumentation (ABBI). Surg. Endosc 1997;11:393-396

20. Marti WR, Zuber M, Oertli D, et al. Advanced Breast Biopsy Instrumentation for the evaluation of impalpable lesions: a reliable diagnostic tool with little therapeutic potential. Eur. J. Surg 2001;167:15-18

21. Burak WE Jr, Owens KE, Tighe MB, et al. Vacuum-assisted stereotactic breast biopsy: histologic underestimation of malignant lesions. Arch. Surg 2000;135:700-703 
22. Pandelidis S, Heiland D, Jones D, et al. Accuracy of 11-gauge vacuum-assisted core biopsy of mammographic breast lesions. Ann. Surg. Oncol 2003;10:43-47

23. Brem RF, Behrndt VS, Sanow L, et al. Atypical ductal hyperplasia: histologic underestimation of carcinoma in tissue harvested from impalpable breast lesions using 11-gauge stereotactically guided directional vacuum-assisted biopsy. A. J. R. Am. J. Roentgenol 1999; 172:1405-1407

24. Joshi M, Duva-Frissora A, Padmanabhan R, et al. Atypical ductal hyperplasia in stereotactic breast biopsies: enhanced accuracy of diagnosis with the mammotome. Breast J 2001;7:207-213

25. Liberman L, Cohen MA, Dershaw DD, et al. Atypical ductal hyperplasia diagnosed at stereotaxic core biopsy of breast lesions: an indication for surgical biopsy. A.J.R. Am. J. Roentgenol 1995; 164:1111-1113

26. Jackman RJ, Burbank F, Parker SH, et al. Atypical ductal hyperplasia diagnosed at stereotactic breast biopsy: improved reliability with 14gauge, directional, vacuum-assisted biopsy. Radiology 1997;204:485488

27. Darling ML, Smith DN, Lester SC, et al. Atypical ductal hyperplasia and ductal carcinoma in situ as revealed by large-core needle breast biopsy: results of surgical excision. A.J.R. J. Am. J. Roentgenol 2000; $175: 1341-1346$

28. Winchester DJ, Bernstein JR, Jeske JM, et al. Upstaging of atypical ductal hyperplasia after vacuum-assisted 11-gauge stereotactic core needle biopsy. Arch. Surg 2003;138:619-623

29. Plantade R, Hammou JC, Fighiera M, et al. Underestimation of breast carcinoma with 11-gauge stereotactically guided directional vacuum-assisted biopsy. J. Radiol 2004;85:391-401
30. Arpino G, Allred DC, Mohsin SK, et al. Lobular neoplasia on core-needle biopsy-clinical significance. Cancer 2004;101:242250

31. Chen SC, Yang HR, Hwang TL, et al. Intraoperative ultrasonographically guided excisional biopsy or vacuum-assisted core needle biopsy for nonpalpable breast lesions. Ann. Surg 2003;238. 738-742

32. Sneige N, Lim SC, Whitman GJ, et al. Atypical ductal hyperplasia diagnosis by directional vacuum-assisted stereotactic biopsy of breast microcalcifications: considerations for surgical excision. Am. J. Clin. Pathol 2003;19:248-253

33. Middleton LP, Grant S, Stephens T, et al. Lobular carcinoma in situ diagnosed by core needle biopsy: when should it be excised? Mod. Pathol 2003;16:120-129

34. Bauer VP, Ditkoff BA, Schnabel F, et al. The management of lobular neoplasia identified on percutaneous core breast biopsy. Breast J 2003;9:4-9

35. Renshaw AA, Cartagena N, Derhagopian RP, et al. Lobular neoplasia in breast core needle biopsy specimens is not associated with an increased risk of ductal carcinoma in situ or invasive carcinoma. Am. J. Clin. Pathol 2002;17:797-799

36. Bibeau F, Chateau MC, Masson B. Management of non-palpable breast lesions with vacuum-assisted large core needle biopsies (Mammotome): experience with 560 procedures at the Val d'Aurelle Center. Ann. Pathol 2003;23:582-592

37. Crisi GM, Mandavilli S, Cronin E, et al. Invasive mammary carcinoma after immediate and short-term follow-up for lobular neoplasia on core biopsy. Am. J. Surg. Pathol 2003;27: 325-333 